\title{
LA LECTURA CRÍTICA: UNA MIRADA DESDE EL AULA EN UNA INSTITUCIÓN EDUCATIVA DE TUNJA, COLOMBIA ${ }^{1}$
}

\author{
Diana Carolina Granados Robles \\ Instituto de Enseñanza Media Diversificada INEM de Tunja (Colombia) \\ carolinagra@hotmail.es \\ David Alberto Londoño Vásquez \\ Institución Universitaria de Envigado (Colombia) \\ dalondono@correo.iue.edu.co
}

Recibido: 14/02/2020 - Aprobado: 04/04/2020

DOI: doi.org/10.17533/udea.lyl.n78a12

\begin{abstract}
Resumen: Este artículo presenta los resultados de una investigación cuyo objetivo fue fortalecer los niveles de lectura crítica de estudiantes de grado noveno en una institución educativa pública de Tunja (Colombia) en 2017. Se realizó una prueba diagnóstica, la cual permitió diseñar talleres pedagógicos e implementarlos en las clases. Posteriormente, se compararon los resultados obtenidos en el diagnóstico con la prueba final y se hizo un seguimiento a seis participantes de los 24 que tomaron ambas. Los resultados fueron positivos, dado que los participantes afianzaron sus niveles de lectura crítica y evidenciaron el desarrollo de capacidades frente a las propiedades textuales.
\end{abstract}

Palabras clave: comprensión textual; estudiantes de secundaria; lectura crítica; producción textual; talleres pedagógicos.

1. Este artículo presenta los resultados de la tesis titulada Fortalecimiento de los niveles de lectura críticaconstructivista: Programa de articulación con la media técnica Sena Regional Boyacá e Institución Educativa INEM Carlos Arturo Torres Peña, grado 9-1, de la ciudad de Tunja (Boyacá), la cual fue requisito de la autora para optar al título de Magíster en Pedagogía de la Universidad Santo Tomás (Colombia).

LINGÜÍSTICA Y LITERATURA

297

ISSN 0120-5587

E-ISSN 2422-3174

N. ${ }^{\circ} 78,2020297-319$ 


\title{
CRITICAL READING: AN OVERVIEW FROM THE CLASSROOM IN A HIGH SCHOOL OF TUNJA, COLOMBIA
}

\begin{abstract}
This paper shows the results of an investigation whose objective was to strengthen the levels of critical reading of $9^{\text {th }}$ grade students in a public school in Tunja (Colombia) in 2017. Thus, a diagnostic test was carried out, which allowed the design of pedagogical workshops and the implementation in the lessons. Subsequently, the results obtained in the diagnosis were compared with the final test and six participants of the 24 who took both were followed up. The results were positive given that the participants strengthened their critical reading levels and evidenced the development of capacities against textual properties.
\end{abstract}

Key words: textual comprehension and production; high-school students; critical reading; pedagogical workshops.

\section{Introducción}

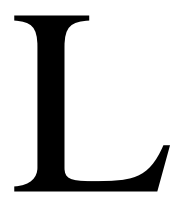

os procesos de lectura y escritura son importantes dentro y fuera del ámbito educativo (Avendaño, 2016; Carrasco, 2019), ya que están inmersos en cualquier contexto en el que se desenvuelve el ser humano (Villalón, 2014; Oxenham, 2017); es así, que existen investigaciones que han efectuado avances en estos procesos, ya sea para inferir (Graham, Gisllepie, \& McKeown, 2013; Holguin, Castillo \& Fabris, 2017) o comprender los textos escritos (Goatly, 2013; González \& Londoño, 2019).

A nivel internacional, han habido trabajos que se centran en la lectura y el desarrollo de habilidades donde se proponen estrategias necesarias para ayudar a formar un pensamiento crítico y reflexivo en los estudiantes (Street, 2014; Iñigo \& Rio, 2017). Estas investigaciones muestran aspectos importantes a tener en cuenta como son: el comprender el propósito lingüístico (Carrasco et al., 2013; Willinsky, 2017), sin dejar de lado un componente fundamental como el contexto (Moreno, García \& Guzman, 2017; Axelsson, Lundqvist, \& Sandberg, 2019), el cual fija una posición al interpretar un texto y propende por la creación de estrategias metacognitivas que van a favorecer los procesos de comprensión y producción textual (Mayoral, Timoneda \& Pérez, 2013; Jiménez, Alvarado \& Calaforra, 2018).

A nivel nacional, se han realizado investigaciones que muestran una dificultad por parte de los estudiantes al argumentar (Barragán \& Arias, 2014; Causado, Santos \& Calderon, 2015), resumir y captar ideas principales (Méndez et al., 2014; Pérez \& La Cruz, 2014), interpretar 
textos escritos (Patiño, 2014; Suárez, Vélez \& Londoño, 2019), así como la falta de formación docente en cuanto a buenas prácticas lectoras (Valverde, 2014; Arellano, 2019).

Y, por último, a nivel regional, se develaron algunas herramientas significativas para la comprensión lectora (Pinzón, Montaña \& Zapata, 2017; Pinto \& Rojas, 2019) y los procesos escriturales (Granados, 2018; Rincón, 2019), donde juega un papel importante la motivación del estudiante al momento de acercarse a la comprensión y la producción textual en el proceso de construcción de ciudadanos críticos, autónomos y democráticos (Romero, 2014; González \& Londoño, 2019).

Por otra parte, la institución educativa (IE) donde se realizó esta investigación está localizada en Tunja (Colombia) donde se han presentado dificultades en comprensión, interpretación y producción textual en los últimos 15 años, lo cual influye notablemente en el rendimiento académico de sus estudiantes, así como en los bajos resultados en las pruebas censales, como figura en el ranking de colegios de Boyacá 2017, donde ocupa el puesto 105 de 412 instituciones educativas, mientras que a nivel nacional, para ese mismo año, ocupó el puesto 916 de 969 con una puntuación en lectura crítica promedio de 56,94 en lectura crítica. Estos resultados, además, pueden relacionarse con la necesidad de desarrollar habilidades para la construcción de competencias ciudadanas y participativas, donde se requiere develar manipulaciones discursivas tan presentes en los textos cuyos objetivos pretenden hegemonizar.

Esta situación hizo necesario que se planteara una investigación que permitiera describir los niveles de lectura crítica de los estudiantes y se evaluaran posibles alternativas para afianzar dichos niveles. De allí, que las categorías teóricas propuestas fueron la lectura crítica y el rendimiento académico.

La lectura crítica propende por una comprensión exigente (Causado, Santos \& Calderon, 2015), que no implica solamente el entender el texto, sino que pretende agregar significados a lo leído mediante la interpretación (Spears \& Spears, 2006; Cassany \& Castellà, 2011). Asimismo, se tiene en cuenta el contexto y las subjetividades tanto del autor como del intérprete (Goatly, 2013; Cardona \& Londoño, 2016), pues a partir de estos se pueden generar múltiples comprensiones (Holguin et al., 2017; Pinto et al., 2019), las cuales estarían directamente conectadas con los acontecimientos vivenciales (Méndez et al., 2014; Wilson, 2016). 
De esta manera, la lectura crítica requiere de un conocimiento de la lingüística textual (Renkema, 2009; Dressler, 2014), donde aspectos como la sintaxis (Song, 2014; Borer, 2017), la semántica (Bondi \& Scott, 2010) y la pragmática (Leech, 2016; Grundy, 2019) juegan un rol esencial para la apropiación del sentido del texto (Montealegre, 2004; Vessey, 2009). Además, se torna esencial la identificación y el análisis de las propiedades textuales, tales como la aceptabilidad, la adecuación, la cohesión, la coherencia, la informatividad, la intencionalidad, la intertextualidad y la situacionalidad comunicativa (Beaugrande \& Dressler, 1981; Souza, 2017), las cuales permiten que el lector pueda dilucidar los propósitos comunicativos (Jiménez et al., 2018;), los géneros textuales (Avendaño, 2016; Navarro \& Aparicio, 2018) y las manipulaciones discursivas (Oxenham, 2017; Jiménez, Riquelme \& Londoño, 2020).

En cuanto al rendimiento académico, es necesario resaltar que usualmente, la valoración que se hace en las investigaciones responde a una perspectiva cuantitativa, la cual se reduce a unos promedios obtenidos por los estudiantes (Rodríguez, Fita \& Torrado, 2004; Fajardo et al., 2017). Esto permite inferir que las posibles causas de los diferentes rendimientos académicos se correlacionen con factores previamente señalados, como el capital cultural presente en la familia, el contexto, el consumo sociocultural y los niveles de comprensión y producción textual (Chaparro, González \& Caso, 2016; Henao \& Londoño, 2018).

Este último factor es significativo, puesto que, en la medida que los estudiantes cuentan con mayor capacidad de comprender, interpretar y producir textos en los ámbitos escolares, su posibilidad de acceso y el procesamiento de la información, la aplicación de diferentes herramientas metacognitivas, la capacidad de asociación conceptual y la construcción de habilidades argumentativas y propositivas se afianzan (Barragán \& Arias, 2014; Axelsson et al., 2019), permitiendo que el estudiante pueda acceder a los diferentes escenarios propuestos en los momentos evaluativos (Wentzel \& Wigfield, 1998; Muriel, Gómez \& Londoño, 2020).

Por eso, se propone un punto de encuentro entre ambas categorías teóricas, pues en la medida que el estudiante alcance a desarrollar mejores niveles de lectura crítica, este no solo podrá distinguir los diferentes elementos textuales, discursivos y argumentativos relacionados con el texto (Cassany, 2003; Castañeda \& Henao, 2011), sino que además podrá ir más allá, centrándose en las valoraciones contextualizadas en las vivencias personales o las condiciones socioculturales, los elementos ideológicos y aspectos dogmáticos (Serrano \& Madrid, 2007; 
Avendaño, 2016), permitiendo afianzar la comprensión textual y producir textos que respondan a los requerimientos académicos definidos (Renkema, 2004; Willinsky, 2017).

\section{Metodología}

El enfoque de la investigación realizada fue cualitativo (Vasilachis, 2006; Denzin \& Lincoln, 2008), de perspectiva hermenéutico-comprensiva (Vessey, 2009; Ángel \& Herrera, 2011) y apoyada en estadística descriptiva (Castañeda \& Henao, 2011; Londoño, Olave, Jaime \& Losada, 2018). Los participantes en esta investigación fueron 24 estudiantes matriculados en el curso de noveno grado de una IE pública de Tunja (Colombia) en 2017. Los jóvenes estaban entre los 14 y 18 años de edad y pertenecían a los estratos 1 y 2 (condiciones socioeconómicas bajas).

Se seleccionó el grado noveno teniendo en cuenta que los estudiantes en Colombia presentan una prueba censal donde la lectura crítica figura entre una de las competencias a evaluar. De igual forma, se escogió esta IE por su influencia en la ciudad de Tunja y debido al alto grado de estudiantes de bajos recursos económicos que se encuentran matriculados en dicha Institución. Además, los niveles de formación alcanzados por sus padres son prioritariamente bachillerato (67\%) y algunos no realizaron estudios $(25 \%)$. Además, no cuentan con hábitos de consumo cultural.

Los 24 participantes presentaron dos pruebas: Una diagnóstica (o de entrada) y otra de salida. Estas pruebas fueron diseñadas según los requerimientos del Ministerio de Educación Nacional MEN de Colombia para el grado noveno en relación a lectura crítica. Ambas pruebas fueron validadas (Vandergrift et al., 2006; Denzin \& Lincoln, 2008) por expertos (2 nacionales y 1 internacional). ${ }^{2}$ La prueba de salida se realizó al finalizar el cuarto trimestre académico, es decir, 9 meses después a la prueba diagnóstica.

Después de la prueba diagnóstica, se seleccionaron a seis de los 24 participantes. La selección fue de forma intencional (Vasilachis, 2006; López, 2004). En esta ocasión, el grupo de escogidos estuvo conformado por tres hombres y tres mujeres, quienes obtuvieron un desempeño

2. Los tres expertos cuentan con formación doctoral y publicaciones en el área del lenguaje.

LINGÜÍSTICA Y LITERATURA

301

ISSN 0120-5587

E-ISSN 2422-3174

N. ${ }^{\circ} 78,2020297-319$ 
alto, medio y bajo en la prueba diagnóstica. ${ }^{3}$ El motivo de esta selección fue posibilitar el seguimiento de dichos participantes en otros dos momentos de la investigación: la intervención realizada a través de talleres pedagógicos.

En el transcurso de los nueve meses que duró la intervención, se realizaron cuatro talleres pedagógicos. El primer taller se centró en la lectura Las maravillas de la voluntad, de Octavio Paz. Con base en ella, los participantes debían elaborar un resumen utilizando las macrorreglas propuestas por van Dijk (1983). De este modo, estos debían identificar el género discursivo del texto y responder unas preguntas. El segundo giró alrededor del texto El pensamiento para un buen vivir de Pepe Mujica, de Ramón Rivas. Allí, los participantes realizaron una lectura crítica según las 22 técnicas propuestas por Cassany (2003). El tercero contó con dos caricaturas de Mafalda, las cuales estaban relacionadas con dos temas socio-políticos en Colombia: el proceso de paz y las elecciones presidenciales. A partir de las mismas, los participantes debían observarlas y luego contestar una serie de preguntas. Finalmente, el cuarto taller se compuso de una caricatura de Matador, el cual tuvo como objetivo trabajar en el nivel inferencial de tipo intratextual, deduciendo mensajes implícitos dentro de la caricatura y respondiendo a las valoraciones subjetivas de cada uno.

\section{Resultados y discusión}

A continuación, se presentarán los resultados obtenidos después del análisis de la unidad recolectada y de las discusiones propuestas a partir de dicho análisis.

\subsection{Pruebas diagnósticas y de salida}

Las pruebas diagnósticas y de salida permitieron evaluar seis categorías relacionadas con la lectura crítica con propósitos académicos (Castañeda \& Henao, 2011; Henao \& Londoño, 2018): el empleo de las macrorreglas en la elaboración del resumen, los niveles de cohesión y

3. Los resultados de la prueba diagnóstica en el curso fueron los siguientes: el $25 \%$ obtuvo una valoración esperada; el $45 \%$, una aceptable; y el $30 \%$, una insuficiente. 
coherencia, la comprensión de los significados, el uso de marcadores discursivos, la conceptualización de la lectura crítica y los niveles de ortografía y puntuación.

\subsubsection{Empleo de las macrorreglas en la elaboración del resumen}

Dentro de la prueba de diagnóstica tomada por los 24 estudiantes del grado noveno, se evaluó la implementación de las macrorreglas en la elaboración del resumen. Su desempeño se valoró en esperado, aceptable e insuficiente.

Los resultados diagnósticos obtenidos fueron del $38 \%$ en insuficiente y del $62 \%$ en aceptable. Esto evidencia la dificultad que tenían los estudiantes en cuanto a la utilización de técnicas adecuadas para producir un resumen (Morales, Cassany, Díaz \& España, 2015; Cardona \& Londoño, 2016), pues en algunos casos no comprendieron el texto suministrado en la prueba diagnóstica, ni tampoco realizaron un adecuado proceso en cuanto a la reescritura del mismo, sobre la información más importante que en él podían encontrar (Spears \& Spears, 2006; Souza, 2017).

En cuanto a los resultados de la prueba de salida, se logró evidenciar un avance significativo: las valoraciones insuficiente y aceptable se redujeron del $38 \%$ al $21 \%$ y del $62 \%$ al $46 \%$, respectivamente. Por ende, la valoración esperada subió de $0 \%$ a un $33 \%$. Por consiguiente, es factible afirmar que el trabajo en clase a partir de la aplicación de las macrorreglas en el resumen (van Dijk, 1983; Montes, Rangel \& Reyes, 2014) ayudó a los participantes a entender la funcionalidad de un resumen frente a la posibilidad de la producción de textos que permitan aprehender las ideas propuestas y consolidarse de manera concisa, coherente y cohesionada (Beaugrande \& Dressler, 1981; Dressler 2014). Ahora, los resultados no son los inicialmente deseados, puesto que todavía hay una tercera parte de los participantes con valoraciones en insuficiente.

\subsubsection{Niveles de cohesión y coherencia}

Frente a la cohesión, los resultados diagnósticos son más positivos, con un $75 \%$ en aceptable y un $25 \%$ en insuficiente. En la prueba de salida, se reducen al $63 \%$ y $13 \%$, respectivamente, 
permitiendo una mejoría frente a un $24 \%$ de porcentaje esperado. En relación a esta propiedad textual, se identificó una dificultad al entrelazar las ideas que se encuentran en el texto, y por lo tanto, se detectó una baja capacidad de jerarquizar ideas, desconociendo la relación que existe entre una y otra (Bondi \& Scott, 2010; Carrasco et al., 2013). La repetición es una constante en los participantes, lo cual hace presumir que no cuentan con un vocabulario suficiente que les permita usar sinónimos (Oxenham, 2017; Souza, 2017) o pronombres (Torres \& Boces, 2012; Song, 2014) en los momentos que son requeridos. Los participantes toman sus escritos, no como una unidad, sino de forma parcializada, pues sus oraciones se desconectan unas con otras, lo que impide reconocer la intensión y el propósito textual (Castañeda \& Henao, 2011; Moreno et al., 2017).

En cuanto a la coherencia, los resultados diagnósticos fueron los mismos de la cohesión. No obstante, los resultados varían en la prueba de salida: el insuficiente se redujo del $25 \%$ al $13 \%$ y el aceptable del $75 \%$ al $67 \%$. Además, el valor esperado sube del $0 \%$ al $20 \%$, indicando una leve mejoría frente a la cohesión. Es importante mencionar la estrecha relación existente entre la coherencia y la cohesión en los procesos escriturales (Louwerse, 2004; Idrobo, 2015).

De igual forma, se estableció que los participantes, si bien tuvieron mejorías, todavía presentaban dificultades en la construcción discursiva tanto en la estructura textual como en los aspectos semánticos, especialmente, cuando se hace un análisis desde lo contextual (Bondi \& Scott, 2010; Cassany \& Castellà, 2011), lo cual puede ser subsanado en el proceso por la participación y acompañamiento de docentes significativos o tutores de escritura (Molina, 2014; Chois et al., 2017).

\subsubsection{Comprensión de los significados}

En relación a este apartado, en las pruebas diagnósticas se detectó que la comprensión de los significados en la elaboración del resumen es mayor en las demás categorías que componen este análisis, con un $79 \%$ en aceptable y un $21 \%$ en insuficiente. Por otro lado, frente a la prueba de salida, los resultados variaron positivamente: la valoración esperada a un $25 \%$, la aceptable, al $67 \%$ y la insuficiente, al $8 \%$. 
Los resultados aún suscitan preguntas frente a la capacidad de los participantes en conectar lo que leen con sus vivencias, su cultura, los aspectos sociales que los rodean (Romero, 2014; Muriel et al., 2020); es decir, hacer el proceso de comprensión una actividad significativa, construir los significados (Luke \& Freebody, 1997; Luke; 2018). Este énfasis en la comprensión y la producción de significados, incluye la activación del conocimiento previo (Tobias, 1994; Irrazabal, 2019), la construcción literal e inferencial de conceptos y procesos (Montealegre, 2004; Holguin et al., 2017), la comparación del texto con otros ejemplos del discurso (Morales et al., 2015; Navarro \& Aparicio, 2018), entre otros.

\subsubsection{Uso de marcadores discursivos}

El uso de los marcadores en el resumen contrasta frente a los aspectos anteriores. El nivel insuficiente fue del $71 \%$ y el aceptable del $29 \%$ en la prueba diagnóstica. No obstante, estos porcentajes cambiaron en la prueba de salida: $46 \%$ y $17 \%$, respectivamente. Además, la valoración esperada fue del $37 \%$.

Estos resultados permiten reafirmar que los participantes no conectan sus ideas, especialmente, a través de marcadores discursivos. Tal vez por desconocimiento o por otras formas de construcción discursiva como la puntuación en sus textos escritos o la entonación en su habla. No obstante, en los textos académicos, es común que se utilicen estos marcadores (Renkema, 2004; Suárez et al., 2019), los cuales están encargados de organizar la información contenida en el texto (van Dijk, 1983; Halliday, 2001), dando cohesión, coherencia y concordancia (Villalón, 2014; Souza, 2017). Afortunadamente, los resultados obtenidos fueron propositivos frente al bajo nivel inicial.

\subsubsection{Conceptualización de la lectura crítica}

En la prueba diagnóstica, los resultados fueron los presupuestados: el $92 \%$ de los participantes no lograron conceptualizar la lectura crítica, mientras que solo el $8 \%$ lo hizo parcialmente. En la prueba de entrada, este panorama mejoró considerablemente. El insuficiente se redujo al $42 \%$, el aceptable aumentó al $38 \%$ y el esperado surgió con un $18 \%$; es decir, un 
$56 \%$ de los participantes pudo interiorizar de qué se trata la lectura crítica (Cassany, 2003; Granados, 2018).

Frente a estos resultados, es necesario resaltar que en la prueba diagnóstica las respuestas más comunes apuntaron a una lectura lineal, literal, puntual, por lo cual no incluían un proceso de producción textual en respuesta a las reacciones generadas. Esto sugiere que para los participantes, la lectura crítica es solo un proceso de lectura y no les demanda acciones diferentes a lo que usualmente hacen cuando leen. En otras palabras, no piensan en el autor y sus subjetividades, el género discursivo del texto, el público al que está dirigido, ni mucho menos reflexionan sobre sus propias reacciones como lectores o evalúan la calidad de las fuentes utilizadas.

\subsubsection{Niveles de ortografía y puntuación}

Los niveles de ortografía y puntuación se clasifican por el número de errores cometidos por cada participante tanto en la prueba diagnóstica como de salida. En relación a la ortografía, los resultados obtenidos inicialmente fueron del $16 \%$ como el nivel esperado (cero errores), de un $16 \%$ en nivel aceptable ( 1 a 5 errores), y del $68 \%$ en nivel insuficiente (más de 5 errores). En esta ocasión, los resultados diagnósticos fueron mayores a los de la prueba de salida (17 \%, 8 \% y $75 \%$, respectivamente). Por otro lado, en relación a la puntuación, en la prueba diagnóstica, los porcentajes obtenidos fueron del $42 \%$ en nivel aceptable ( 1 a 5 errores), y del $58 \%$ en nivel insuficiente (más de 5 errores). En la prueba de salida, varían levemente los porcentajes: $46 \%$ y $50 \%$, respectivamente, dejando un nivel esperado correspondiente al $4 \%$.

Ante estos porcentajes, es importante mencionar que si bien los resultados de las pruebas de salida no son ni levemente aceptables frente a los valores esperados, el número de palabras escritas por prueba pasó de 246 a 483, es decir, los textos producidos se hicieron más extensos en las pruebas de salida, lo que explica el aumento del porcentaje de errores.

Con respecto a los errores ortográficos, los más frecuentes fueron las omisiones, las transposiciones, las conjugaciones verbales, los acentos y las diéresis, los usos de la h y de los nombres propios. Estos tipos de errores pueden catalogarse como de tipo natural y arbitrario (Gómez \& Gómez, 2015; Sotomayor et al., 2017). En la puntuación, los participantes tuvieron 
dificultades para indicar pausas y realizar entonaciones. Además, se evidenció una dificultad en la identificación de la coma y el punto. En ese sentido, ninguno de los participantes utilizó el punto y coma, donde hubiese sido posible, aunque en la mayoría de estos casos lo reemplazaron por un punto seguido (Borer, 2017; Holguin, 2017).

\subsection{Talleres pedagógicos}

Como se mencionó anteriormente, se realizaron cuatro talleres pedagógicos al grupo en un transcurso de 9 meses. A continuación se presentan los resultados de los seis participantes seleccionados para este momento.

\subsubsection{Primer taller}

El primer taller giró alrededor de la lectura Las maravillas de la voluntad, de Octavio Paz. A partir de ella, los participantes elaboraron un resumen empleando las macrorreglas (van Dijk, 1983). Asimismo, estos identificaron el género discursivo de la lectura y respondieron a unas preguntas de comprensión lectora. A continuación se indican los resultados obtenidos por los participantes, en este taller:

\begin{tabular}{lllllll}
\hline Taller $\mathbf{N}^{\circ} \mathbf{1}$ & $\mathbf{M 1}$ & $\mathbf{M} 2$ & $\mathbf{M 3}$ & $\mathbf{H 1}$ & $\mathbf{H 2}$ & $\mathbf{H 3}$ \\
\hline Insuficiente & $87 \%$ & $14 \%$ & $54 \%$ & $80 \%$ & $73 \%$ & $66 \%$ \\
\hline Aceptable & $0 \%$ & $73 \%$ & $33 \%$ & $13 \%$ & $20 \%$ & $27 \%$ \\
\hline Esperado & $13 \%$ & $13 \%$ & $13 \%$ & $7 \%$ & $7 \%$ & $7 \%$ \\
\hline
\end{tabular}

Tabla 1. Resultados porcentuales de cada participante en el taller pedagógico $n .^{\circ} 1$. Fuente: Elaboración propia.

En el primer taller se evidencia un ligero avance de los participantes, pues se pueden apreciar valoraciones de lo esperado. La estudiante M1 es la participante que mayor porcentaje obtuvo en insuficiente, seguido de H1. Estos dos participantes son los dos con menor desempeño en la 
prueba diagnóstica. De forma consecutiva, el mejor desempeño lo lograron las estudiantes M2 y M3.

En este taller se logra develar que con respecto a la elaboración del resumen hay avances; aunque, todavía se identifican confusiones frente a qué son y cómo se usan las macrorreglas (la supresión, la generalización y la construcción), pues lo que en algunos casos sucede, es que los participantes seleccionan oraciones que para ellos son importantes y las yuxtaponen literalmente, sin revisar los niveles de cohesión y coherencia (Castañeda \& Henao, 2011; Morales et al., 2015).

No obstante, en estos resúmenes, los participantes lograron interpretar y comprender (Gadamer \& Hahn, 1997; Vessey, 2009) palabras que encuentran en la lectura, dándoles un significado a partir de la inferencia (Holguin, 2017; Pinto \& Rojas, 2019), lo que permite evidenciar pautas de la lectura crítica, como el propósito del autor a través del texto y las construcciones discursivas (Cassany, 2003; Serrano \& Madrid, 2007).

\subsubsection{Segundo taller}

El segundo taller se diseñó con base en el texto El pensamiento para un buen vivir de Pepe Mujica, del Ramón Rivas. La actividad propuesta consistió en la elaboración de una lectura crítica según las 22 técnicas propuestas por Cassany (2003). A continuación se indican los resultados obtenidos por los participantes, en este taller:

\begin{tabular}{lllllll}
\hline Taller N $\mathbf{N}^{\circ} \mathbf{1}$ & $\mathbf{M 1}$ & $\mathbf{M 2}$ & $\mathbf{M 3}$ & $\mathbf{H 1}$ & $\mathbf{H 2}$ & $\mathbf{H 3}$ \\
\hline Insuficiente & $35 \%$ & $5 \%$ & $48 \%$ & $57 \%$ & $83 \%$ & $74 \%$ \\
\hline Aceptable & $56 \%$ & $43 \%$ & $22 \%$ & $43 \%$ & $13 \%$ & $22 \%$ \\
\hline Esperado & $9 \%$ & $52 \%$ & $30 \%$ & $0 \%$ & $4 \%$ & $4 \%$ \\
\hline
\end{tabular}

Tabla 2. Resultados porcentuales de cada participante en el taller pedagógico $n .^{\circ} 2$. Fuente: Elaboración propia.

Como se puede observar en los resultados, los estudiantes M1, M2, M3 y H1 muestran un avance significativo en la disminución del nivel de desempeño insuficiente. Caso contrario 
ocurre con los estudiantes $\mathrm{H} 2$ y H3, quienes alcanzaron un nivel de desempeño insuficiente en el segundo taller.

En el caso de $\mathrm{H} 2$ y H3, si bien se identifica una mejoría en las propiedades textuales (Beaugrande \& Dressler, 1981; Dressler, 2014), no hay una apropiación temática de lo propuesto por la lectura (Renkema, 2004; Grundy, 2019). En otras palabras, los participantes siguen quedándose en una lectura lineal y literal (Villalón, 2014; Suárez et al., 2019). Además, no contextualizan el texto frente a los aspectos sociales y culturales (Méndez et al., 2014; Henao y Londoño, 2018). De allí, que su lectura se asemeje a un parafraseo y no permita dilucidar los aportes de dichos participantes (Luke, 2019; Jiménez et al., 2020).

\subsubsection{Tercer taller}

El tercer taller se centró en 2 caricaturas de Mafalda, a las cuales los participantes analizaron. Estas caricaturas estaban relacionadas con el proceso de paz y las elecciones presidenciales. A continuación se indican los resultados obtenidos por los participantes, en este taller:

\begin{tabular}{lllllll}
\hline Taller N $^{\circ} \mathbf{1}$ & $\mathbf{M 1}$ & $\mathbf{M} 2$ & $\mathbf{M 3}$ & $\mathbf{H 1}$ & $\mathbf{H 2}$ & H3 \\
\hline Insuficiente & $13 \%$ & $0 \%$ & $0 \%$ & $37 \%$ & $0 \%$ & $0 \%$ \\
\hline Aceptable & $87 \%$ & $38 \%$ & $87 \%$ & $38 \%$ & $100 \%$ & $38 \%$ \\
\hline Esperado & $0 \%$ & $62 \%$ & $13 \%$ & $25 \%$ & $0 \%$ & $62 \%$ \\
\hline
\end{tabular}

Tabla 3. Resultados porcentuales de cada participante en el taller pedagógico $n$. $^{\circ}$. Fuente:

Elaboración propia.

Tal como se muestra en la tabla anterior, hubo un avance en los niveles de desempeño de los estudiantes objeto de estudio, en los casos como M2, M3, H2 y H3. Además, se observa que desaparece el nivel de desempeño insuficiente y se avanza a los niveles de desempeño aceptable y esperado, lo cual es positivo para esta investigación. Con respecto al nivel esperado, los estudiantes M1 y H2 no lograron obtenerlo.

Lo anterior evidencia la capacidad de análisis que han alcanzado los participantes con los talleres pedagógicos aplicados, lo cual indica, a su vez, que las caricaturas resultan una buena 
herramienta que ayuda a afianzar el proceso de lectura crítica. Además, la comprensión de lectura implica fijar una posición crítica y con argumentos claros acerca de un tema leído para el descubrimiento de lo oculto (Parodi, Peronard \& Ibáñez, 2020; Ibáñez, 2012). De igual forma, se proporciona una posición donde se esgrimen argumentos válidos acerca de la caricatura suministrada, ya que en ellos muestran su ideología a través de sus escritos, acerca de temas de actualidad (Wilson, 2016; Luke, 2019). Asimismo, sus respuestas demuestran una capacidad de síntesis, pues sus oraciones unen elementos relevantes que dan un sentido global del texto (van Dijk, 1983; Souza, 2017).

\subsubsection{Cuarto taller}

El cuarto y último taller se compuso de una caricatura de Matador. Allí, los participantes realizaron inferencias de tipo intratextual, deduciendo mensajes implícitos dentro de la caricatura y respondiendo a las valoraciones subjetivas de cada uno. A continuación se indican los resultados obtenidos por los participantes, en este taller:

\begin{tabular}{lllllll}
\hline Taller $\mathbf{N}^{\circ} \mathbf{1}$ & $\mathbf{M 1}$ & $\mathbf{M 2}$ & $\mathbf{M 3}$ & $\mathbf{H 1}$ & $\mathbf{H 2}$ & $\mathbf{H 3}$ \\
\hline Insuficiente & $7 \%$ & $0 \%$ & $0 \%$ & $29 \%$ & $4 \%$ & $3 \%$ \\
\hline Aceptable & $78 \%$ & $40 \%$ & $70 \%$ & $40 \%$ & $82 \%$ & $30 \%$ \\
\hline Esperado & $15 \%$ & $60 \%$ & $30 \%$ & $31 \%$ & $14 \%$ & $67 \%$ \\
\hline
\end{tabular}

Tabla 4. Resultados porcentuales de cada participante en el taller pedagógico $n .{ }^{\circ} 4$. Fuente:

Elaboración propia.

Lo anterior permite aseverar que la utilización de los talleres pedagógicos ayudó a mejorar el nivel de lectura de los participantes, dado que cada uno de ellos tuvo la oportunidad de avanzar a su ritmo (Pérez \& La Cruz, 2014; Muriel et al., 2020) y de concertar sus capacidades en el proceso de lectura crítica (Avendaño, 2016; Cardona \& Londoño, 2016), permitiendo potenciar sus propias competencias relacionadas con la comprensión y producción textual (Castañeda \& Henao, 2011; Molina, 2014), enfocadas en un objetivo, enriqueciendo su vocabulario, la capacidad de relacionar información nueva con la conocida, realizando inferencias y 
construyendo argumentos cuyos garantes cumplen con elementos de idoneidad, eludiendo la elaboración de falacias argumentativas, en la mayoría de los casos (Cassany, 2003; Bondi \& Scott, 2010). En cuanto a la caricatura, buscaron sobre la situación que llevó al autor a plasmarla, pues lo consideraron importante para responder las preguntas propuestas (Axelsson et al., 2019). El hecho de leer el texto como una unidad permitió a los participantes una mejor comprensión del mismo (Oxenham, 2017; Irrazabal, 2019).

\section{Conclusiones}

Las IE en Colombia han estado realizando diferentes actividades pedagógicas en pro de afianzar los niveles de lectura crítica en sus estudiantes; puesto que entienden la existencia de una relación significativa entre los procesos de comprensión y producción textual mediados en los ámbitos académicos, así como del desarrollo de competencias que empoderan a dichos estudiantes frente a su rol como ciudadanos desde una perspectiva de indagación, reflexión, decisión y participación, y del rendimiento académico en las diferentes disciplinas que componen la formación.

Sin embargo, los resultados censales indican que estos esfuerzos no son óptimos. Por eso, fue necesario repensar la forma cómo se venía trabajando la lectura crítica en esta institución educativa pública en Tunja, (Colombia) especialmente, con los estudiantes de noveno grado, los cuales participan de una medición censal al finalizar el año, terminando la formación de básica secundaria.

Esta propuesta pedagógica llegó a ser significativa gracias a la información recolectada en la prueba diagnóstica, la cual permitió identificar los elementos que requerían de mayor cuidado en proceso de formación de los estudiantes y potenciarlos. Además, la perspectiva sociocultural asumida en la intervención facilitó el diseño de actividades en clase, aprovechando el conocimiento previo de los participantes, sus intereses y sus vivencias.

No obstante, es importante resaltar que los resultados obtenidos en la prueba de salida no fueron completamente satisfactorios, dado que aún se presentaban valoraciones en nivel insuficiente. Esto responde a los niveles de ingreso de los participantes y a cómo asumieron su rol en los diferentes talleres pedagógicos realizados puesto que, en algunos casos, los 
participantes que obtuvieron un desempeño aceptable inicialmente estuvieron por encima de quienes tuvieron un rendimiento considerado como deseado, especialmente, si se comparan las mujeres con los hombres.

Finalmente, el potencial de una investigación de este tipo es significativo por la posibilidad de conocer a los estudiantes, identificar sus capacidades y los niveles de sus competencias frente al proceso de formación, ya que al tener claras las dificultades y sus potenciales, las propuestas pedagógicas responden a un contexto y a una situación definida, propendiendo por mejores prácticas pedagógicas, clases más significativas, estrategias didácticas más funcionales y estudiantes mejor educados.

\section{Referencias bibliográficas}

1. Ángel, D. \& Herrera, J. D. (2011). La propuesta hermenéutica como crítica y como criterio del problema del método. Estudios de Filosofía, 43, 9-29. Recuperado de http://revistas.udea.edu.co/revistas/index.php/estudios_de_filosofia/article/view/11565

2. Arellano, P. E. (2019). La construcción del rol docente desde una mirada renovadora de la lectura y la escritura. Pedagogía y Saberes, 51, 23-32. Disponible en: https://revistas.pedagogica.edu.co/index.php/PYS/article/view/10236

3. Avendaño, G. S. (2016). La lectura crítica en Educación Básica Secundaria y Media: la voz de los docentes. Cuadernos de Lingüística Hispánica, 28, 207-232.

4. Axelsson, A., Lundqvist, J., \& Sandberg, G. (2019). Influential Factors on Children's Reading and Writing Development: The Perspective of Parents in a Swedish Context. Early Child Development and Care, 1-13. Recuperado de https://www.tandfonline.com/doi/full/10.1080/03004430.2019.1590348

5. Barragán, F. E. \& Arias, A. D. (2014). Dilemas morales para contextos rurales: Construcción y validación en básica secundaria de una institución educativa colombiana. Revista Electrónica Educare, $\quad$ 18(2), 95-116. Recuperado de https://www.scielo.sa.cr/scielo.php?script=sci_arttext\&pid=S1409-42582014000200005

6. Beaugrande, R. \& Dressler, W. U. (1981). Introduction to Text Linguistics. Routledge.

7. Bondi, M. \& Scott, M. (Eds.). (2010). Keyness in Texts (Vol. 41). John Benjamins Publishing. 
8. Borer, H. (2017). Morphology and Syntax. The Handbook of Morphology, 149-190. Recuperado de https://onlinelibrary.wiley.com/doi/abs/10.1002/9781405166348.ch8

9. Cardona, P. A. \& Londoño, D. A. (2016). El sentido de la lectura crítica en contexto. Katharsis, 22, 375-401. Recuperado de http://revistas.iue.edu.co/index.php/katharsis/article/view/835

10. Carrasco, A. (2019). De la escuela a la universidad: las prácticas de lectura y escritura en el último año de la secundaria. Letras, 8. Recuperado de https://www.perio.unlp.edu.ar/ojs/index.php/letras/article/view/5780/5007

11. Carrasco, A., Encinas, M. T., Castro A, M. \& López, G. (2013). Lectura y escritura académica en la educación media superior y superior. Revista Mexicana de Investigación Educativa, 18(57), 349-354. Recuperado de http://www.scielo.org.mx/scielo.php?pid=S140566662013000200002\&script=sci_arttext

12. Cassany, D. (2003). Aproximaciones a la lectura crítica: teoría, ejemplos y reflexiones. Tarbiya: Revista de Investigación e Innovación Educativa del Instituto Universitario de Ciencias de la Educación, 32, 113-32. Recuperado de https://repositori.upf.edu/handle/10230/21224

13. Cassany, D. \& Castellà, J. M. (2011). Aproximación a la literacidad crítica. Perspectiva, 28(2), 353-374. Recuperado de https://repositori.upf.edu/handle/10230/21187

14. Castañeda, L. S. \& Henao, J. I. (2011). La lingüística textual y la cultura escrita en la Universidad. Zona Próxima, 6, 12-31. Recuperado de http://rcientificas.uninorte.edu.co/index.php/zona/article/view/1704/1105

15. Causado, R. E., Santos, B. \& Calderon, I. (2015). Desarrollo del pensamiento crítico en el área de ciencias naturales en una escuela de secundaria. Revista de la Facultad de Ciencias, 4(2), 17-42. Recuperado de https://revistas.unal.edu.co/index.php/rfc/article/view/51437

16. Chaparro, A. A., González, C. \& Caso, J. (2016). Familia y rendimiento académico: configuración de perfiles estudiantiles en secundaria. Revista Electrónica de Investigación Educativa, $\quad$ 18(1), 53-68. Recuperado de http://www.scielo.org.mx/scielo.php?script=sci_arttext\&pid=S1607-40412016000100004

17. Chois, P. M., Casas, A. C., López, A., Prado, D. M. \& Cajas, E. Y. (2017). Percepciones sobre la tutoría entre pares en escritura académica. Magis. Revista Internacional de Investigación en Educación, 9(19), 165-184. 
18. Denzin, N. K., \& Lincoln, Y. S. (2008). Introduction: The discipline and Practice of Qualitative Research. In N. K. Denzin \& Y. S. Lincoln (Eds.), Strategies of Qualitative Inquiry (pp. 1-43). Sage Publications.

19. Dressler, W. U. (Ed.). (2014). Current Trends in Textlinguistics (Vol. 2). Walter de Gruyter $\mathrm{GmbH} \& \mathrm{Co} \mathrm{KG}$.

20. Fajardo, F., Maestre, M., Felipe, E., León del Barco, B. \& Polo, M. I. (2017). Análisis del rendimiento académico de los alumnos de Educación Secundaria Obligatoria según las variables familiares. Educación XXI: Revista de la Facultad de Educación, 20(1), 209-232. Recuperado de http://e-spacio.uned.es/fez/view/bibliuned:EducacionXXI-2017-20-1-5045

21. Fountas, I. C., Pinnell, G. S. \& Le Verrier, R. (2001). Guided Reading. Portsmouth, NH: Heinemann.

22. Gadamer, H. G. \& Hahn, L. E. (1997). The Philosophy of Hans-George Gadamer. Chicago.

23. Goatly, A. (2013). Critical Reading and Writing: An Introductory Coursebook. Routledge.

24. Gómez, A. \& Gómez, M. T. (2015). Escritura ortográfica y mensajes de texto en estudiantes universitarios. Perfiles Educativos, 37(150), 91-104. Recuperado de http://www.scielo.org.mx/scielo.php?script=sci_arttext\&pid=S0185-26982015000400006

25. González, M. C. \& Londoño, D. A. (2019). Estrategias pedagógicas de literacidad: experiencia significativa en una Institución Educativa de Boyacá. Revista Latinoamericana de Ciencias Sociales, Niñez y Juventud, 17(1), 253-268. Recuperado de https://www.redalyc.org/jatsRepo/773/77360021016/77360021016.pdf

26. Graham, S., Gillespie, A., \& McKeown, D. (2013). Writing: Importance, Development, and Instruction. Reading and Writing, 26(1), 1-15. Recuperado de https://link.springer.com/article/10.1007/s11145-012-9395-2

27. Granados, D. C. (2018). Niveles de lectura crítica en la institución educativa INEM Carlos Arturo Torres Peña. Experiencias Investigativas y Significativas, 4(4), 1-14. Disponible en: http://experiencias.iejuliussieber.edu.co/index.php/Exp-inv/article/view/66

28. Grundy, P. (2019). Doing Pragmatics. Routledge.

29. Halliday, M. A. (2001). Lenguaje como Semiótica Social. México: Fondo de Cultura Económica. 
30. Henao, J. I. \& Londoño, D. A. (2018). Deserción, literacidad y elección de carrera: una revisión desde la mirada de los estudiantes y los profesores de la Facultad de Ciencias Empresariales de la IUE. Aglala, 9(1), 232-262. Recuperado de https://dialnet.unirioja.es/servlet/articulo?codigo=6832768

31. Holguin, J. A., Castillo, R. \& Fabris, M. (2017). Inferencias causales de profesores que realizan la lectura de textos con implicancia causal y subordinación oracional. Fides et RatioRevista de Difusión Cultural y Científica de la Universidad La Salle en Bolivia, 14(14), 75-98. Recuperado de http://www.scielo.org.bo/scielo.php?pid=S2071081X2017000200006\&script=sci_abstract\&tlng=en

32. Ibáñez, R. (2012). La comprensión del discurso escrito: Una propuesta teórico-metodológica para su evaluación. Revista Signos, 45(78), 20-43. Recuperado de https://scielo.conicyt.cl/scielo.php?pid=S0718-09342012000100002\&script=sci_arttext\&tlng=e 33. Idrobo, J. E. (2015). Escritura y pre-escritura. Proyecto LEO.

34. Iñigo, L. \& Rio, V. (2017). Extensión de la escolaridad y obligatoriedad de la escuela secundaria en Argentina: el papel de la universalización de la lectura y escritura. Universitas Humanística, (83), 213-243. Recuperado de http://www.scielo.org.co/scielo.php?pid=S0120$48072017000100213 \&$ script $=$ sci_abstract\&tlng=en

35. Irrazabal, N. (2019). La comprensión de textos expositivos en estudiantes universitarios: la función del conocimiento previo. Revista de Psicología, 6(12), 7-21. Recuperado de http://erevistas.uca.edu.ar/index.php/RPSI/article/viewFile/2481/2310

36. Jiménez, M., Riquelme, A. \& Londoño, D. (2020). Literacidad como promoción del pensamiento crítico en la primera infancia. Educere, 77(24) 117-134. Recuperado de http://epublica.saber.ula.ve/index.php/educere/article/view/15772

37. Jiménez, V., Alvarado, J. M. \& Calaforra, P. J. (2018). Las estrategias metacognitivas aplicadas a la escritura como predictoras de la calidad de la escritura espontánea. Electronic Journal of Research in Education Psychology, 16(45), 301-323. Recuperado de http://ojs.ual.es/ojs/index.php/EJREP/article/view/2095

38. Leech, G. N. (2016). Principles of Pragmatics. Routledge. 
39. Londoño, D., Olave, J., Jaime, J. \& Losada, N. (2018). Lógicas, enfoques y epistemologías sobre educación y pedagogía. En: B. Gallardo-Cerón et al. (Eds.), Educación y Pedagogía. Trayectos recorridos (pp. 96-155). Manizales: Universidad de Manizales.

40. López, P. L. (2004). Población muestra y muestreo. Punto Cero, 9(8), 69-74. Recuperado de http://www.scielo.org.bo/scielo.php?pid=s1815-02762004000100012\&script=sci_arttext

41. Louwerse, M. M. (2004). Un modelo conciso de cohesión en el texto y coherencia en la comprensión. Revista Signos, 37(56), 41-58. Recuperado de https://scielo.conicyt.cl/scielo.php?pid=S0718-09342004005600004\&script=sci_arttext

42. Luke, A. (2018). Critical Literacy in Australia: A Matter of Context and Standpoint. In Critical Literacy, Schooling, and Social Justice (pp. 168-188). Routledge.

43. Luke, A. \& Freebody, P. (1997). Shaping the Social Practices of Reading. Constructing Critical Literacies: Teaching and Learning Textual Practice, 6, 460-475.

44. Mayoral, S., Timoneda, C. \& Pérez, F. (2013). Evaluación de los procesos metacognitivos en estudiantes de Grado en maestro de Educación Infantil y Primaria en tareas de lectura. Aula Abierta, 41(3), 5-12. Recuperado de https://dialnet.unirioja.es/servlet/articulo?codigo=4401086 45. Méndez, J. C., Espinal, C., Vera, D.; Gómez, J. \& Serna, C. (2014). La lectura crítica en la educación superior: un estado de la cuestión. Revista Virtual Universidad Católica del Norte, 41, 4-18. Recuperado de https://www.redalyc.org/pdf/1942/194229980002.pdf

46. Molina, V. (2014). Centros de escritura: una mirada retrospectiva para entender el presente y futuro de estos programas en el contexto latinoamericano. Legenda, 18(18), 9-33.

47. Montealegre, R. (2004). La comprensión del texto: sentido y significado. Revista Latinoamericana de Psicología, 36(2), 243-255. Recuperado de https://www.redalyc.org/pdf/805/80536205.pdf

48. Montes, A. M., Rangel, Y. \& Reyes, J. A. (2014). Comprensión lectora. Noción de lectura y uso de macrorreglas. $R a$ Ximhai, 10(5), 265-277. Recuperado de https://www.redalyc.org/pdf/461/46132134018.pdf

49. Morales, O., Cassany, D., Díaz, N. \& España, L. B. (2015). Resúmenes y abstracts de artículos científicos: variaciones retóricas e implicaciones didácticas. Acta Bioclínica, 5(9), 3346.

Recuperado

de http://www.revencyt.ula.ve/storage/repo/ArchivoDocumento/actabio/v5n9/art05.pdf 
50. Moreno, C., García, E. \& Guzmán, F. (2017). Los hábitos de lectura y escritura en los estudiantes de Educación Primaria: un análisis Dentro y Fuera de la escuela. Porta Linguarum, 2, 117-137. Recuperado

de https://idus.us.es/handle/11441/75224;jsessionid=51E5290AF2B53CA94A071BEFB313CAA9 51. Muriel, L. E., Gómez, L. S. \& Londoño, D. A. (2020). La riqueza de la evaluación en la escuela, un tesoro no oculto. Praxis \& Saber, 11(25), 113-132. Recuperado de https://revistas.uptc.edu.co/index.php/praxis_saber/article/view/9359

52. Navarro, F. \& Aparicio, G. (2018). Didáctica basada en géneros discursivos para la lectura, escritura y oralidad académicas. Manual de lectura, escritura y oralidad académicas para ingresantes a la universidad, 13. Bernal: Universidad Nacional de Quilmes.

53. Oxenham, J. (2017). Literacy: Writing, Reading and Social Organisation. Routledge.

54. Parodi, G., Peronard, M. \& Ibáñez, R. (2010). Saber Leer. Madrid: Aguilar.

55. Patiño, C. (2014). Apuntes para una historia de la educación en Colombia. Actualidades Pedagógicas, $\quad$ 1(64), 261-264. $\quad$ Recuperado de https://ciencia.lasalle.edu.co/cgi/viewcontent.cgi?article=1260\&context=ap

56. Pérez, V. \& La Cruz, A. (2014). Estrategias de enseñanza y aprendizaje de la lectura y escritura en educación primaria. Zona Próxima, 21, 1-16. Recuperado de https://www.redalyc.org/pdf/853/85332835002.pdf

57. Pinnell, G. S., \& Fountas, I. C. (2011). The Continuum of Literacy Learning, Grades 3-8: A Guide to Teaching. Heinemann.

58. Pinto, J. P. \& Rojas, S. P. (2019). Lectura crítica en el aula en relación con dimensiones inferencial y literal. Educación y Ciencia, 23, 265-279. Recuperado de https://revistas.uptc.edu.co/index.php/educacion_y_ciencia/article/view/10284

59. Pinzón, W. D., Montaña, M. D. \& Zapata, L. A. (2017). Lecturalia: una experiencia de mediación y comprensión de lectura en la Escuela Normal Superior Santiago de Tunja. Experiencias Investigativas y Significativas, 3(3), 13-23. Recuperado de http://experiencias.iejuliussieber.edu.co/index.php/Exp-inv/article/view/11 60. Renkema, J. (2004). Introduction to Discourse Studies. John Benjamins Publishing. 
61. Rincón, A. M. (2019). El poder de la información: una propuesta pedagógica para la lectura crítica en aula. Experiencias Investigativas y Significativas, 5(5), 143-154. Recuperado de http://experiencias.iejuliussieber.edu.co/index.php/Exp-inv/article/view/109

62. Rodríguez, S., Fita, E. \& Torrado, M. (2004). El rendimiento académico en la transición secundaria-universidad. Revista de Educación, 34(1), 391-414. Recuperado de http://www.ince.mec.es/revistaeducacion/re334/re334_22.pdf

63. Romero, L. C. (2014). Encontrar alternativas de motivación hacia la lectura y la escritura en la escuela: un reto con frutos perdurables. Experiencias Investigativas y Significativas, 1(1), 135141. Recuperado de http://experiencias.iejuliussieber.edu.co/index.php/Expinv/article/view/85/73

64. Serrano, S., \& Madrid, A. (2007). Competencias de lectura crítica: una propuesta para la reflexión y la práctica. Acción Pedagógica, 16(1), 58-68. Recuperado de https://dialnet.unirioja.es/servlet/articulo?codigo=2968602

65. Song, J. J. (2014). Linguistic Typology: Morphology and Syntax. Routledge.

66. Sotomayor, C., Ávila, N., Bedwell, P., Domínguez, A., Gómez, G. \& Jéldrez, E. (2017). Desempeño ortográfico de estudiantes chilenos: claves para la enseñanza de la ortografía. Estudios Pedagógicos (Valdivia), 43(2), 315-332. Recuperado de https://scielo.conicyt.cl/scielo.php?pid=S0718-07052017000200017\&script=sci_arttext\&tlng=e 67. Souza, S. (2017). A noção de intertextualidade e de interdiscursividade na linguística textual e na análise do discurso: perspectivas. Revista Virtual Lingu@ Nostr@, 5(1), 97-111. Recuperado de http://www.linguanostra.net/index.php/Linguanostra/article/view/87

68. Spears, D. M. \& Spears, D. M. (2006). Developing Critical Reading Skills. New York: McGraw-Hill.

69. Street, B. V. (2014). Social Literacies: Critical Approaches to Literacy in Development, Ethnography and Education. Routledge.

70. Suárez, P. A., Vélez, M. \& Londoño, D. A. (2019). Niveles de literacidad en tercer grado de una institución educativa de Bello. Enunciación, 24(1), 15-28. Recuperado de https://revistas.udistrital.edu.co/index.php/enunc/article/view/13249

71. Tobias, S. (1994). Interest, Prior Knowledge, and Learning. Review of Educational Research, 64(1), 37-54. Recuperado de https://journals.sagepub.com/doi/abs/10.3102/00346543064001037 
72. Torres, C. \& Boces, G. (2012). Reformulación y uso del pronombre demostrativo neutro 'esto’en la elaboración de síntesis escritas por parte de estudiantes universitarios. Revista Signos, 45(79), 198-225. Recuperado de https://scielo.conicyt.cl/scielo.php?pid=S0718$09342012000200005 \&$ script=sci_arttext\&tlng=p

73. Valverde, Y. (2014). Lectura y escritura con sentido y significado, como estrategia de pedagógica en la formación de maestros. Fedumar Pedagogía y Educación, 1. Recuperado de http://editorial.umariana.edu.co/revistas/index.php/fedumar/article/view/453

74. van Dijk, T. A. (1983). La ciencia del texto: un enfoque interdisciplinario. Paidós Ibérica.

75. Vandergrift, L., Goh, C. C., Mareschal, C. J., \& Tafaghodtari, M. H. (2006). The Metacognitive Awareness Listening Questionnaire: Development and Validation. Language Learning, 56(3), 431-462

76. Vasilachis, I. (2006). La investigación cualitativa. En Estrategias de investigación cualitativa (pp. 23-64). Recuperado de http://jbposgrado.org/icuali/investigacion\%20cualitativa.pdf 77. Vessey, D. (2009). Gadamer and the Fusion of Horizons. International Journal of Philosophical Studies, 17(4), 531-542. Recuperado de https://www.tandfonline.com/doi/abs/10.1080/09672550903164459

78. Villalón, M. (2014). Alfabetización inicial: claves de acceso a la lectura y escritura desde los primeros meses de vida. Ediciones UC.

79. Wentzel, K. R. \& Wigfield, A. (1998). Academic and Social Motivational Influences on Students' Academic Performance. Educational Psychology Review, 10(2), 155-175. Recuperado de https://link.springer.com/article/10.1023/A:1022137619834

80. Willinsky, J. (2017). The New Literacy: Redefining Reading and Writing in the Schools. Routledge.

81. Wilson, K. (2016). Critical Reading, Critical Thinking: Delicate Scaffolding in English for Academic Purposes (EAP). Thinking Skills and Creativity, 22, 256-265. Recuperado de https://www.sciencedirect.com/science/article/pii/S1871187116301365 